\title{
Relative Frequencies of Constrained Events in Stochastic Processes: an Analytical Approach
}

\author{
S. Rusconi ${ }^{* 1}$, E. Akhmatskaya ${ }^{1,2}$, D. Sokolovski ${ }^{2,3}$, N. Ballard ${ }^{4}$ and J.C. de la \\ $\mathrm{Cal}^{4}$ \\ ${ }^{1}$ BCAM - Basque Center for Applied Mathematics, Alameda de Mazarredo 14, \\ 48009 Bilbao, Bizkaia, Spain \\ ${ }^{2}$ IKERBASQUE, Basque Foundation for Science, E-48013 Bilbao, Spain \\ ${ }^{3}$ Departmento de Química-Física, Universidad del País Vasco, UPV/EHU, \\ Leioa, Spain \\ ${ }^{4}$ POLYMAT and Grupo de Ingeniería Química, Dpto. de Qumica Aplicada, \\ University of the Basque Country UPV/EHU, Joxe Mari Korta Zentroa, Tolosa \\ Etorbidea 72, 20018 Donostia/San Sebastián, Spain
}

September 23, 2015

\begin{abstract}
Stochastic Simulation Algorithm (SSA) and the corresponding Monte Carlo (MC) method are ones of the most common approaches for studying stochastic processes. They relies on the knowledge of inter-events probability density functions (pdf), and on the information about dependencies between all possible events. Analytical representations of a pdf are difficult to specify in advance, in many real life applications. Knowing a shape of pdf, and using experimental data, different optimization schemes can be applied in order to evaluate the probability density functions and, therefore, the properties of the studied system. Such methods, however, are computationally demanding, and often not feasible. We show that in the case where experimentally accessed properties are directly related to the frequencies of events involved, it may be possible to replace the heavy Monte Carlo core of optimization schemes with an analytical solution. Such replacement not only provides more accurate estimation of the properties of the process, but also reduces the simulation time by a factor of order of the sample size (at least $\approx 10^{4}$ ). The proposed analytical approach is valid for any choice of the pdf. The accuracy, computational efficiency, and advantages of the method over MC procedures, are demonstrated in the exactly solvable case and in evaluation of branching fractions in Controlled Radical Polymerization (CRP) of acrylic monomers. This polymerization can be modelled by a constrained stochastic process. Constrained systems are quite common, and this makes the method useful for various applications.
\end{abstract}

\section{Introduction}

Simulation of stochastic processes is a powerful tool for modelling and describing the evolution of various phenomena in natural and human-made systems. A well known example of such

*srusconi@bcamath.org 
modelling is the Stochastic Simulation Algorithm (SSA) developed by D. Gillespie [1]. In physics community, it is also known as the $n$-fold way, introduced by Bortz et al. [2]. SSA is a Monte Carlo (MC) based method: it draws multiple realizations of the process and then computes statistics on them. From now on, we will refer to SSA as MC method. This approach is based on the assumption that the studied system is well-mixed, and also memoryless. These assumptions lead to independent exponentially distributed inter-event times probability density functions [3]. As intuition suggests, this set of hypothesis does not hold for all phenomena of practical interest. One such example is a constrained stochastic process, where the occurrence of some events may depend on the previous history of the process (see [4] for examples of constrained stochastic processes). In this cases, the dependencies can be realized either explicitly by introducing constraints in the SSA algorithm, or implicitly, through a modification of interevent times probability density functions (pdf).

Either way, well posed formulations of stochastic processes require the knowledge of inter-event times probability density functions. One can assume a particular functional shape for these pdf, but meaningful values of the pdf parameters are also needed, in order to complete the description of the model and run the corresponding Monte Carlo simulation. In practice, appropriate pdf parameters are difficult to identify.

One possible way to estimate the unknown parameters is to employ an optimisation scheme, which uses available experimental data. The idea behind a fitting scheme is to build a cost function $J$, which measures the disagreement between the experimental data, and the data obtained by the proposed MC method. A fitting scheme seeks to minimize the cost function $J$, in order to find the set of pdf parameters which gives the best agreement with the experimental data.

Since the stochastic simulation is a part of the cost function evaluation, multiple runs are needed until a good fitting is obtained. Thus, regardless of the choice of the optimization route, and of the particular cost function, any MC based fitting scheme is computationally expensive.

In this paper we derive an analytical approach for evaluation of $J$, as a function of pdf's parameters, without running the corresponding $\mathrm{MC}$ simulation. The method is free of the statistical errors affecting Monte Carlo based simulations. It can be incorporated into fitting schemes used to study various phenomena for which the experimentally observed properties are directly related to frequencies of the events involved. Examples of such phenomena include branching rate reduction in Controlled Radical Polymerization [5], high-frequency pulsed laser polymerization of acrylates [6], reduction in rate of polymerization in RAFT-mediated polymerizations [7, 8], and the absence of chain transfer to PVA in SET-DTLRP of PVC in aqueous media [9].

Our goal is to provide analytical expressions for the asymptotic frequencies of events involved in constrained stochastic processes, thus avoiding costly Monte Carlo evaluations, used in a fitting scheme. A natural application for the new method is the computation of branching fractions in Controlled Radical Polymerization (CRP) of acrylic monomers [10].

The paper is organized as follows. In section 2 we propose a general approach for calculation of asymptotic relative frequencies of events in constrained stochastic processes. It is solved analytically in section 3 for a process with a single constraint, and the results are compared with the ones earlier provided in [11]. CRP and the formation of branches in CRP of acrylates is briefly described in section 4. In subsection 4.1 the MC fitting method earlier proposed for evaluation of the branching fraction in the CRP [12] is summarized. Our alternative analytical approach is developed in subsection 4.2. The results of comparison between two different methodologies are provided in subsection 4.3. The concluding remarks are given in section 5 . 


\section{Relative Asymptotic Frequencies of Events in Constrained Stochastic Processes}

Consider a stochastic process with a given total number of events $n_{T}>>1$. These events correspond to the realizations of the random variables building the stochastic process. Each event may have $N$ possible outcomes. A particular outcome is given by a possible value assumed by the random variables. The outcomes are constrained, i.e. the outcome $i=1, . ., N$ can occur if and only if at least $c_{i j}$ outcomes $j=1, \ldots, N$ have already occurred after the previous occurrence of the $i$-th outcome.

Our objective is to compute ratios between asymptotic numbers of occurred outcomes, $n_{i}$ and $n_{j}$, corresponding to two events of different types, $i$ and $j$.

It is worthy to remark that there are the conditions the system needs to satisfy in order to be able to evolve its state. First, each outcome must not be constrained by itself, or

$$
c_{i i}=0 \quad \forall i=\{1, . ., N\},
$$

and second, there is at least one outcome free to occur, or (s.t. is a shorthand for "such that")

$$
\exists i \in\{1, . ., N\} \quad \text { s.t. } \quad c_{i j}=0 \forall j=\{1, . ., N\} .
$$

Otherwise, if $c_{i j} \neq 0 \wedge c_{j i} \neq 0$ for all $i, j$, none of events are possible.

The $n_{T}$ events can be partitioned as follows. Let us divide all events into $2^{N}$ non-overlapping subsets labelled $\left(j_{1}, j_{2}, . ., j_{N}\right)$, where $j_{k}=0,1$ and $k=1,2, . ., N . j_{k}=1$ means that the $k$-th outcome is possible, whereas if $j_{k}=0$ it is not possible due to constraints. Let $n\left(j_{1}, j_{2}, . ., j_{N}\right)$ be the number of events in a subset, e.g.,

$$
n(1,0, \ldots, 0)
$$

is the number of events for which the outcome $i=1$ is possible, but the rest of them are not. Obviously

$$
\sum_{j_{1}, . ., j_{N}=0,1} n\left(j_{1}, j_{2}, . ., j_{N}\right)=n_{T} .
$$

Let $n_{i}\left(j_{1}, j_{2}, . ., j_{N}\right)$ be the number of outcomes of a kind $i$ in the subset $\left(j_{1}, j_{2}, . ., j_{N}\right)$. The assumption is that the events belonging to each subset $\left(j_{1}, j_{2}, . ., j_{N}\right)$ are independent and identically distributed (i.i.d.). Such an assumption does not lead to loss of generality because the constraints used in building different subsets can contain the information about the dependencies between events [13].

Then if $n\left(j_{1}, j_{2}, . ., j_{N}\right) \rightarrow+\infty$, the probability $\mathbb{P}_{i}\left(j_{1}, j_{2}, . ., j_{N}\right)$ for the $i$-th outcome to occur in the set $\left(j_{1}, j_{2}, . ., j_{N}\right)$ corresponds to the limit of frequency of the $i$-th outcome, i.e.:

$$
\mathbb{P}_{i}\left(j_{1}, j_{2}, . ., j_{N}\right)=\frac{n_{i}\left(j_{1}, j_{2}, . ., j_{N}\right)}{n\left(j_{1}, j_{2}, . ., j_{N}\right)} \quad \forall i \in\{1, . ., N\}
$$

A recent formulation of the Gillespie Stochastic Simulation Algorithm (SSA) [3] can be used to define the probability $\mathbb{P}_{i}\left(j_{1}, j_{2}, . ., j_{N}\right)$. Let independent random variables $T_{1}, T_{2}, . ., T_{N}$ be the times required for the next occurrence of the respective outcome. The SSA suggests to pick the outcome that realizes the minimal occurrence time among the possible ones. In other words, 


$$
\mathbb{P}_{i}\left(j_{1}, j_{2}, . ., j_{N}\right)= \begin{cases}0 & \text { if } j_{i}=0 \\ \mathbb{P}\left(T_{i}<T_{k}: \forall k \neq i \text { s.t. } j_{k}=1\right) & \text { if } j_{i}=1 \wedge \exists k \neq i \text { s.t. } j_{k}=1 \\ 1 & \text { if } j_{i}=1 \wedge j_{k}=0 \forall k \neq i\end{cases}
$$

Equations (5) and (6) offer the way for calculation of the asymptotic numbers of outcomes of a kind $i, n_{i}$, for any $i$ :

$$
n_{i}=\sum_{j_{1}, . ., j_{N}} \mathbb{P}_{i}\left(j_{1}, j_{2}, . ., j_{N}\right) n\left(j_{1}, j_{2}, . ., j_{N}\right) \quad \forall i \in\{1, . ., N\} .
$$

The ratio between $n_{i}$ and $n_{k}$ for any $i$ and $k$ can be immediately obtained from (7) and (6). The number of possible outcomes $N$ should not be crucial for the proposed approach. In particular, the proposed solution holds for big values of $N$. More important for the applicability of the method are the possible dependencies between different events. In general, complex dependencies between different subsets may lead to non-converged asymptotic behaviour of the ratios of interest. However, when the constraints are limited and well defined, as in the examples presented in the following sections, the ratios of interest can be evaluated exactly for any value of $N$.

\section{A Process with a Single Constraint}

Before applying the suggested methodology to a fitting procedure outlined in section 1 we test it on the simple model introduced in [11]. We consider the case of a stochastic process with only two possible outcomes, 1 and 2. The first one is free to occur with the occurrence rate $c_{1}$, but the second one must wait till at least $n_{0}$ occurrences of kind 1 after its own previous occurrence. Its occurrence rate is $c_{2}$. There are only two possible subsets in this case (the order of events is preserved as described above):

$$
(1,0),(1,1) \text {. }
$$

The corresponding probabilities are given by

$$
\begin{aligned}
& \mathbb{P}_{1}(1,0)=1, \quad \mathbb{P}_{1}(1,1)=\mathbb{P}\left(T_{1}<T_{2}\right), \\
& \mathbb{P}_{2}(1,0)=0, \quad \mathbb{P}_{2}(1,1)=\mathbb{P}\left(T_{2}<T_{1}\right) .
\end{aligned}
$$

The total number of events $n(1,0)$ in the subset $(1,0)$ is given by $n_{0}$ outcomes 1 for each occurrence of the outcome 2 :

$$
n(1,0)=n_{2} n_{0},
$$

where $n_{2}$ is the asymptotic total number of outcomes 2 . The total number of events in the complementary subset $n(1,1)$ can be computed by subtraction:

$$
n(1,1)=n_{T}-n_{2} n_{0} .
$$

Equation 7 gives

$$
n_{1}=n_{2} n_{0}+\mathbb{P}\left(T_{1}<T_{2}\right)\left(n_{T}-n_{2} n_{0}\right),
$$




$$
n_{2}=\mathbb{P}\left(T_{2}<T_{1}\right)\left(n_{T}-n_{2} n_{0}\right),
$$

where $T_{1}$ and $T_{2}$ are the times required to fire the next occurrences of kind 1 and 2 respectively. Equations (12) and (13) can be rewritten as

$$
\begin{gathered}
n_{1}=\frac{\mathbb{P}\left(T_{1}<T_{2}\right)+n_{0} \mathbb{P}\left(T_{2}<T_{1}\right)}{1+n_{0} \mathbb{P}\left(T_{2}<T_{1}\right)} n_{T}, \\
n_{2}=\frac{\mathbb{P}\left(T_{2}<T_{1}\right)}{1+n_{0} \mathbb{P}\left(T_{2}<T_{1}\right)} n_{T} .
\end{gathered}
$$

Equations (14) and (15) show that as $n_{T} \rightarrow \infty$, the asymptotic fraction $n_{2} / n_{1}$ reaches the fixed asymptotic value. In particular

$$
\frac{n_{2}}{n_{1}}=\frac{\mathbb{P}\left(T_{2}<T_{1}\right)}{\mathbb{P}\left(T_{1}<T_{2}\right)+n_{0} \mathbb{P}\left(T_{2}<T_{1}\right)} .
$$

Following [3], we assign independent exponentially distributed probability density functions to the random variables $T_{1}$ and $T_{2}$ :

$$
T_{1} \stackrel{\text { ind. }}{\sim} \operatorname{Exp}\left(c_{1}\right), \quad T_{2} \stackrel{\text { ind. }}{\sim} \operatorname{Exp}\left(c_{2}\right) .
$$

The probabilities in (16) can be computed as suggested in Appendix A, which gives

$$
\mathbb{P}\left(T_{1}<T_{2}\right)=\frac{c_{1}}{c_{1}+c_{2}}, \quad \mathbb{P}\left(T_{2}<T_{1}\right)=\frac{c_{2}}{c_{1}+c_{2}} .
$$

Thus, the asymptotic ratio between the number of outcomes 2 and the number of outcomes 1 becomes

$$
\frac{n_{2}}{n_{1}}=\frac{c_{2}}{c_{1}+n_{0} c_{2}} .
$$

This result confirms the validity of the analytical approach presented for this model in [11]. It is also in good agreement with the data obtained using the Monte Carlo method proposed in [11], as is shown in Figure 1.

\section{Controlled Radical Polymerization}

In this section we introduce a real phenomenon that can be studied by the proposed analytical method. In particular, we are interested in a polymerization process known as Controlled Radical Polymerization (CRP) of acrylic monomers [10].

Radical polymerization is a method by which high molecular weight polymer molecules can be formed by successive addition of individual monomer units. In radical polymerization of vinyl monomers, each addition, or propagation, outcome regenerates the active radical species at the chain end, and the chain continues to grow until it terminates, typically by reaction with another radical.

Alternatively, the reactive radical at the end of the chain can be transferred to a carbon atom within the chain, thus generating a so called mid-chain radical. In radical polymerization of acrylic monomers this is particularly important and it occurs via a process (outcome) known as backbiting. Due to the specific molecular orientation required for the backbiting outcome to occur, at least three propagation outcomes must take place before the next backbiting outcome 


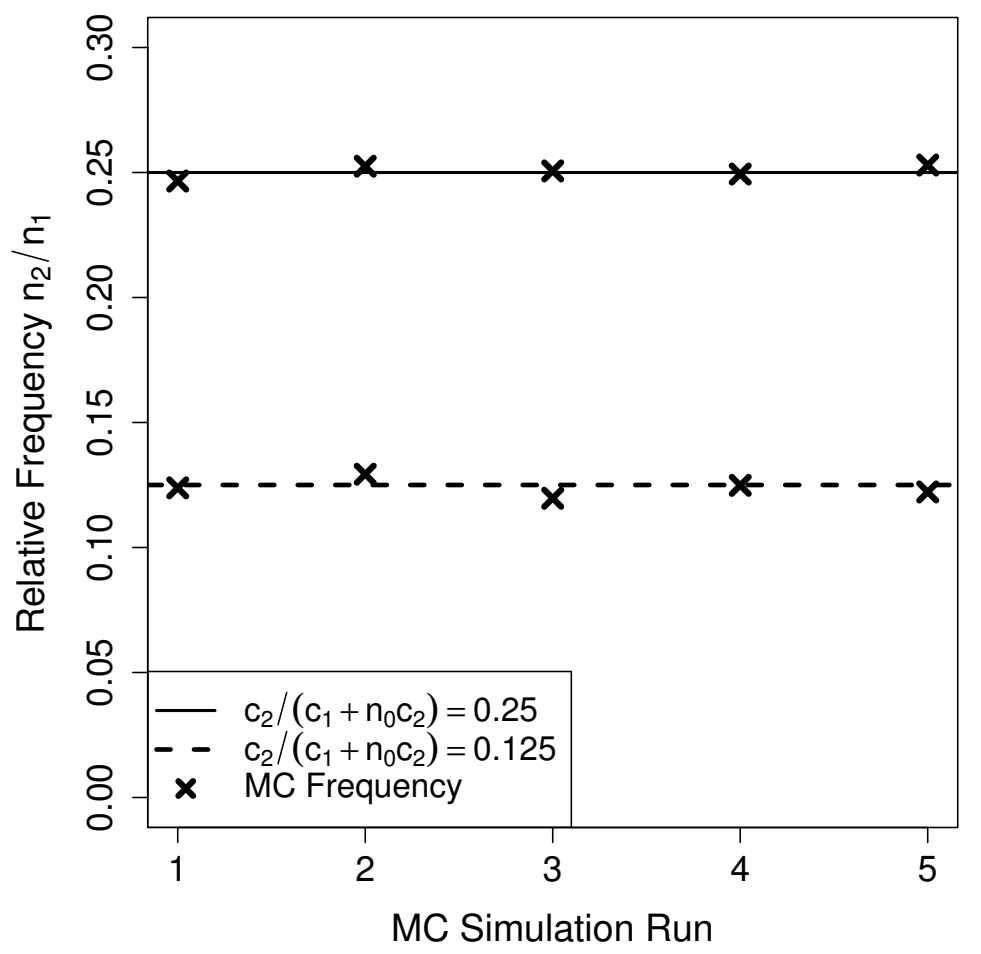

Figure 1: Comparison between the analytical solution (19) (lines) and corresponding statistics (crosses) obtained by the Monte Carlo (MC) method proposed in [11]. Five independent runs are performed for two different parameters set: $n_{0}=3, c_{2} / c_{1}=1$ (solid line) and $n_{0}=3$, $c_{2} / c_{1}=0.2$ (dashed line). The MC sample size is equal to $G=10^{4}$.

occurs.

Addition of monomer units to the mid-chain radical, which is formed by backbiting, results in the formation of a branched structure. Obtaining information about the kinetics of the process, and the relative rate of reactions between backbiting and propagation, are important for understanding polymerization of acrylic monomers. The ratio of the two competitive outcomes has a strong impact on polymer micro-structure and the mechanical properties of the resulting polymer. It can be measured by evaluating the branching fraction, which is determined experimentally as the ratio of the number of branches compared to the number of propagation outcomes that have occurred.

Controlled Radical Polymerization (CRP) is a special type of radical polymerization which is conducted in the presence of an additional chemical reagent known as a control agent. In CRP the reactive radical chain end is subjected to frequent deactivation and reactivation steps allowing to control molecular weight of the polymer chain. Reversible deactivation of the reactive chain end is an outcome which occurs in competition with propagation and backbiting. We refer to it as the deactivation outcome.

Although classical chemical reaction kinetics dictate that the imposition of the additional competitive process of chain deactivation should not impact on the ratio of backbitings to propaga- 
tions, experimental evidence has shown that there is a strong reduction in branching fraction under CRP conditions $[5,12,14]$. In order to explain this reduction the existence of a nonexponential probability density function has been proposed [12]. In [12], a Monte Carlo fitting scheme was suggested for evaluation of the appropriate parameters of the non-exponential pdf in order to fit the experimental branching fractions. The data provided in [12], shown in Figure 2, will be used for comparing our analytical approach with the MC based procedure. Two sets of data with corresponding uncertainty intervals were obtained by two different experimental procedures, known as bulk and solution polymerization. Each polymerization was conducted at different control agent concentrations, thus giving a range of data points for the fitting procedure.

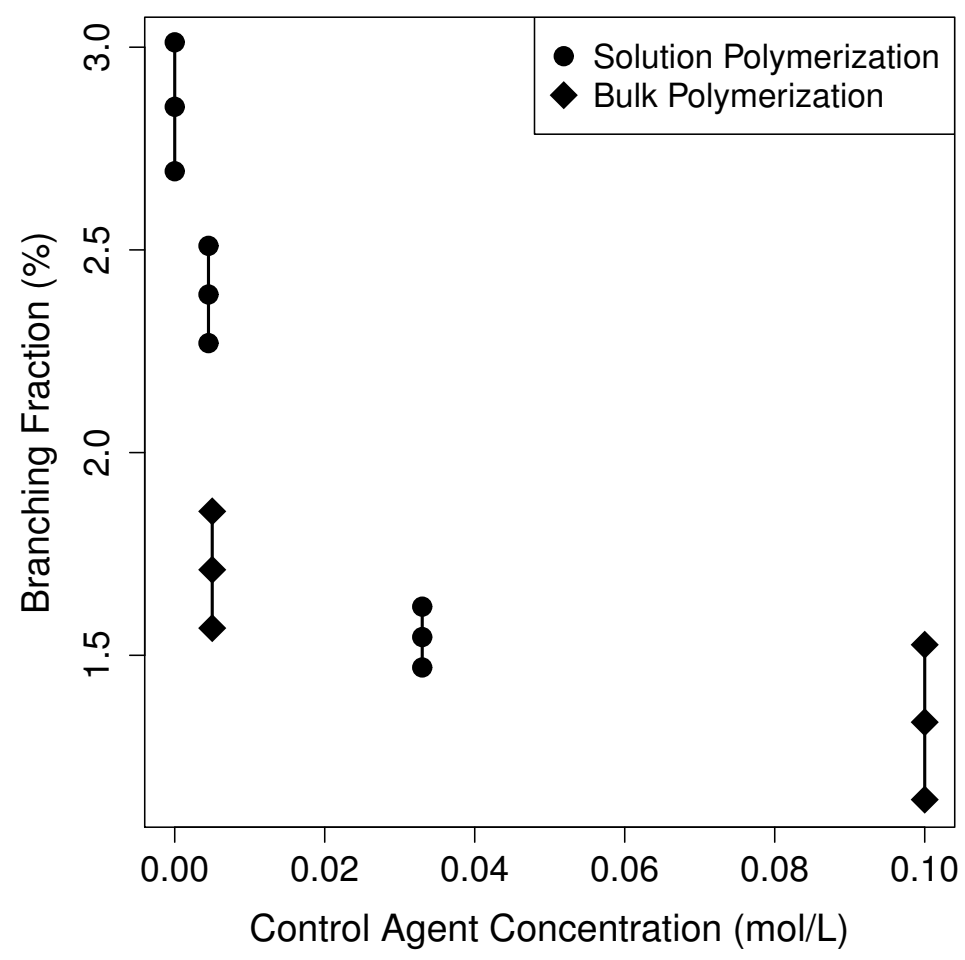

Figure 2: Experimental branching fractions and corresponding uncertainty intervals [12].

\subsection{The Monte Carlo Fitting Scheme}

The Monte Carlo Fitting Scheme for evaluation of the branching fractions [12] combines a MC method with an optimization algorithm.

The MC method follows the SSA [3], where independent linear exponential pdf, $\operatorname{Linexp}\left(b_{i}, \tau_{i}\right)$, are assumed for required times for each outcome. It means that the time required for a propagation is $T_{p} \stackrel{\text { ind. }}{\sim} \operatorname{Linexp}\left(b_{p}, \tau_{p}\right)$, for a backbiting is $T_{r} \stackrel{\text { ind. }}{\sim} \operatorname{Linexp}\left(b_{r}, \tau_{r}\right)$ and for a deactivation is $T_{d} \stackrel{\text { ind. }}{\sim} \operatorname{Linexp}\left(b_{d}, \tau_{d}\right)$. The linear exponential pdf is defined as: 


$$
T_{i} \sim \operatorname{Linexp}\left(b_{i}, \tau_{i}\right) \Leftrightarrow f_{L E}\left(t ; b_{i}, \tau_{i}\right)=\left\{\begin{array}{ll}
\frac{2}{b_{i}^{2}+2 b_{i} \tau_{i}} t & \text { if } 0 \leq t<b_{i} \\
\frac{2 b_{i}}{b_{i}^{2}+2 b_{i} \tau_{i}} e^{-\left(\frac{t-b_{i}}{\tau_{i}}\right)} & \text { if } t \geq b_{i}
\end{array} .\right.
$$

Different concentrations of monomers and control agents are considered as it is proposed in the supporting information of [12]. The MC method is summarized in Algorithm algorithm 1.

The optimization routine can be described as follows:

1. Choose the values of the parameters $\left\{b_{p}, \tau_{p}, b_{r}, \tau_{r}, b_{d}, \tau_{d}\right\}$ for a particular attempt of the chosen optimization algorithm.

2. Run the MC method proposed in Algorithm algorithm 1 and calculate the mean ratio between numbers of backbitings and propagations, $r$, corresponding to the current choice of parameters.

3. Choose and compute the cost function $J$. The middle point of each uncertainty interval is used.

4. Restart from item 1 until $J$ is minimized.

We have to stress that for the purposes of this paper the choice of an optimization algorithm is not important. However, in practice one should go for the most efficient one available, since this would help to reduce the number of Monte Carlo runs required for finding the optimal parameters. Next we show how the MC algorithm in the optimization scheme described above can be replaced with a significantly more effective analytical approach.

The reason to invoke Monte Carlo procedure is because it is one of the most commonly used method to study such processes. Thus, we began by defining the kinetics of the system in terms of Monte Carlo rules, or by defining the explicitly constrained pdf proposed in algorithm 1 . Next we show how to directly solve the dynamics of the system without having to resort to $\mathrm{MC}$ approach. 


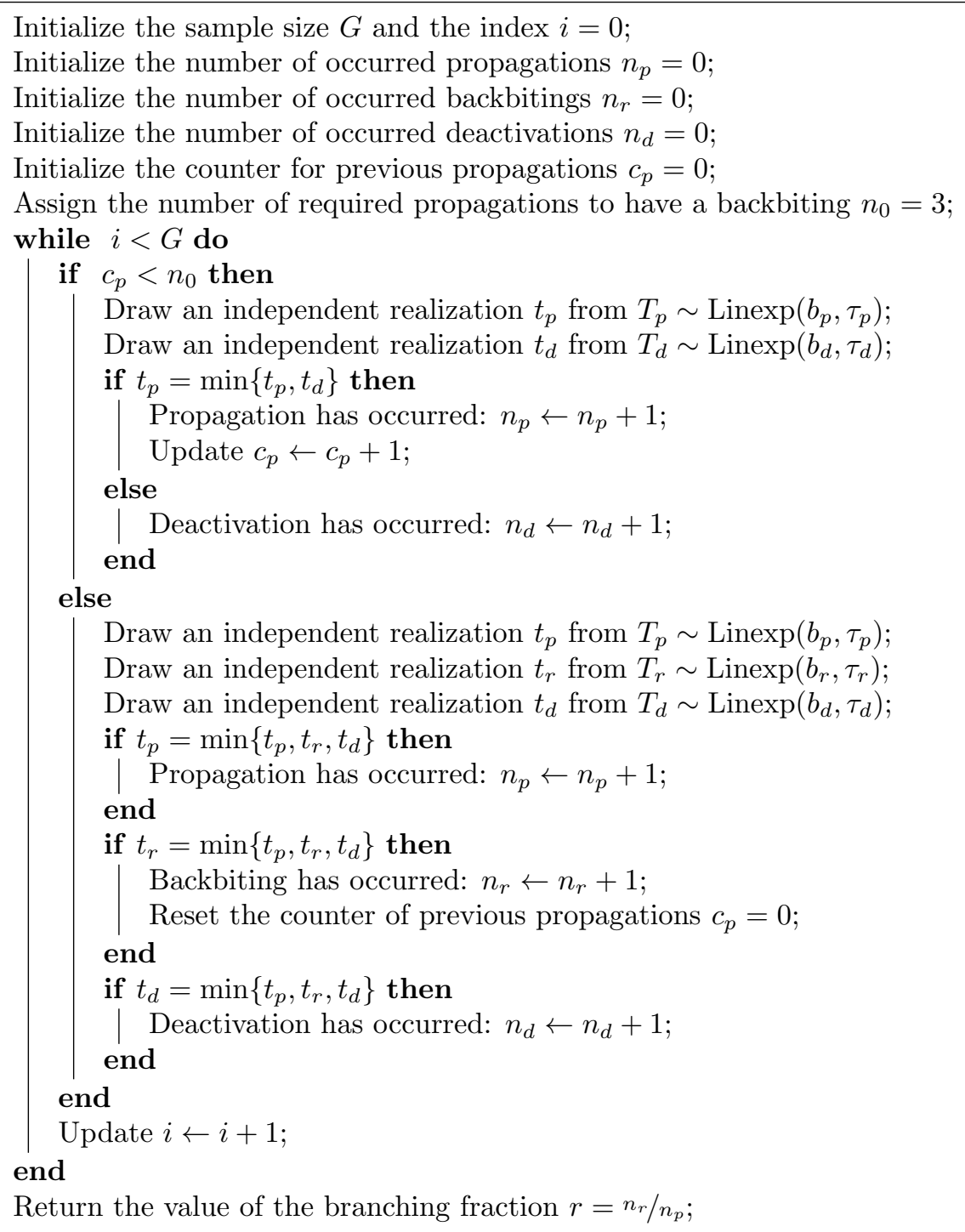

Algorithm 1: The MC method for simulation of the evolution of Controlled Radical Polymerization carried out in the presence of control agent. The method returns the final branching fraction of the created chain.

\subsection{Analytical Expressions for Asymptotic Relative Frequencies of Events in CRP}

We will follow the ideas described in section 2. In particular, let $n_{p}, n_{d}$ and $n_{r}$ respectively be the asymptotic mean number of occurred propagations, deactivations and backbitings. Then the branching fraction can be calculated as a ratio between $n_{r}$ and $n_{p}$ using equations (6) and (7). As stated in section 4, propagation and deactivation are always possible, whereas backbiting needs at least $n_{0}=3$ previous propagations to occur. Hence, we have two subsets (the order is: propagation $(\mathrm{p})$, deactivation $(\mathrm{d})$, backbiting $(\mathrm{r}))$ : 


$$
(1,1,0),(1,1,1) \text {. }
$$

The following are the probabilities for each outcome in each subset:

$$
\begin{aligned}
\mathbb{P}_{p}(1,1,0)=\mathbb{P}\left(T_{p}<T_{d}\right), & \mathbb{P}_{p}(1,1,1)=\mathbb{P}\left(T_{p}<T_{d}, T_{r}\right), \\
\mathbb{P}_{d}(1,1,0)=\mathbb{P}\left(T_{d}<T_{p}\right), & \mathbb{P}_{d}(1,1,1)=\mathbb{P}\left(T_{d}<T_{p}, T_{r}\right), \\
\mathbb{P}_{r}(1,1,0)=0, & \mathbb{P}_{r}(1,1,1)=\mathbb{P}\left(T_{r}<T_{p}, T_{d}\right) .
\end{aligned}
$$

The total number of events that cannot be a backbiting, $n(1,1,0)$, is given by $n_{0}$ propagations for each occurred backbiting, and by the number of deactivations occurred when backbiting is not possible:

$$
n(1,1,0)=n_{0} n_{r}+n_{d}(1,1,0)=n_{0} n_{r}+\mathbb{P}\left(T_{d}<T_{p}\right) n(1,1,0) .
$$

Equation 23 can be rewritten as

$$
n(1,1,0)=\frac{n_{0} n_{r}}{\mathbb{P}\left(T_{p}<T_{d}\right)} .
$$

Apparently,

$$
n(1,1,1)=n_{T}-n(1,1,0) .
$$

Then, Equation 7 yields

$$
\begin{gathered}
n_{p}=\mathbb{P}\left(T_{p}<T_{d}\right) n(1,1,0)+\mathbb{P}\left(T_{p}<T_{d}, T_{r}\right)\left[n_{T}-n(1,1,0)\right], \\
n_{d}=\mathbb{P}\left(T_{d}<T_{p}\right) n(1,1,0)+\mathbb{P}\left(T_{d}<T_{p}, T_{r}\right)\left[n_{T}-n(1,1,0)\right], \\
n_{r}=\mathbb{P}\left(T_{r}<T_{p}, T_{d}\right)\left[n_{T}-n(1,1,0)\right] .
\end{gathered}
$$

From Eqs. (26) and (28) we have:

$$
\begin{gathered}
n_{r}=\frac{\mathbb{P}\left(T_{p}<T_{d}\right) \mathbb{P}\left(T_{r}<T_{p}, T_{d}\right)}{\mathbb{P}\left(T_{p}<T_{d}\right)+n_{0} \mathbb{P}\left(T_{r}<T_{p}, T_{d}\right)} n_{T}, \\
n_{p}=\frac{\mathbb{P}\left(T_{p}<T_{d}\right)\left[\mathbb{P}\left(T_{p}<T_{d}, T_{r}\right)+n_{0} \mathbb{P}\left(T_{r}<T_{p}, T_{d}\right)\right]}{\mathbb{P}\left(T_{p}<T_{d}\right)+n_{0} \mathbb{P}\left(T_{r}<T_{p}, T_{d}\right)} n_{T} .
\end{gathered}
$$

Equations (29) and (30) show that as $n_{T} \rightarrow \infty$, the branching fraction $r=n_{r} / n_{p}$ reaches the fixed asymptotic value. In particular, the ratio $r$ is a function of probabilities involving the random variables $T_{p}, T_{d}$ and $T_{r}$ only.

This implies two important consequences: (i) our solution holds for any choice of inter-events times pdf and (ii) it is possible to express the branching fraction as a function of the pdf parameters, once the probabilities of interest are computed. We explain how to compute the required probabilities for a generic case in Appendix A. This method is still valid for the linear exponential case (20) considered in this study.

The proposed analytical approach is based on the assumption that the asymptotic limit is reached. Thus, it describes the statistics of the asymptotic states of a stochastic process. Reaching the asymptotic limit via Gillespie type of simulations is costly, since also the transient 
dynamics need to be performed. Availability of analytical shortcuts avoids having to deal with fluctuations present in finite systems. In particular, in the considered application (CRP), the reaction continues until all the reactants are used up. For this reason, the our method agrees with the experimental data for a reaction which may be considered to have reached its asymptotic state.

\subsection{Numerical Results}

We present the results of numerical tests run for validation of the proposed approach. The experimental data used in the tests were described in section 4 .

In Figure 3, we assess the accuracy provided by two fitting approaches for calculation of the branching fractions using the experimental observations. The first approach (the data are shown by open circles) corresponds to the optimization routine explained in subsection 4.1. The second method (crosses) follows the same optimization route (Nelder-Mead method [15]) and evaluates the same cost function $J$, but the Monte Carlo evaluation of the branching fractions is replaced with the analytical expressions given in (29), (30). Both, the MC method and the analytical approach, use a linear exponential inter-events time pdf (20). As it follows from Figure 3, the sample size $G=10^{4}$ in the Monte Carlo approach guarantees the same level of accuracy provided by the analytical method. Table 1 shows the optimized parameters for the linear exponential inter-events time pdf (20).

Although both methods can offer comparable accuracies, it is not the case for the computational cost. The optimization routine performed with the analytical approach is up to $10^{4}$ times faster than the one using the MC method of the same level of accuracy (MC sample size $G=10^{4}$ ). Comparative computational times are shown in Figure 4.

It is clear that the degree of speed up provided by the analytical approach over the MC method is determined by the MC sample size $G$. Indeed, the computational complexity of the analytical method is $O(1)$ whereas it is $O(G)$ in the case of Monte Carlo. This is confirmed by the numerical tests.

Different optimization algorithms and inter-events time pdf were tested. In Figure 5, Figure 6 and Table 2 we present the results obtained with the fitting scheme in which both the optimization routine and the inter-events time pdf differ from those applied in the previous test. In particular, a Genetic Algorithm [16] has been selected for optimization. Pure exponential pdf was assigned for propagation and deactivation, whereas linear exponential pdf (20) was chosen for backbiting. The rationale behind the choice of the pdf for propagation and deactivation is confirmed by our previous test, described above, which results in very small optimal parameters $b_{p}$ and $b_{d}$. This suggests that the optimal pdf choices for propagation and deactivation are very close to an exponential function. The results shown in Figure 5 justify this choice. Also, these results confirm that the proposed analytical approach is valid for various choices of optimization routines and inter-events time pdf. 


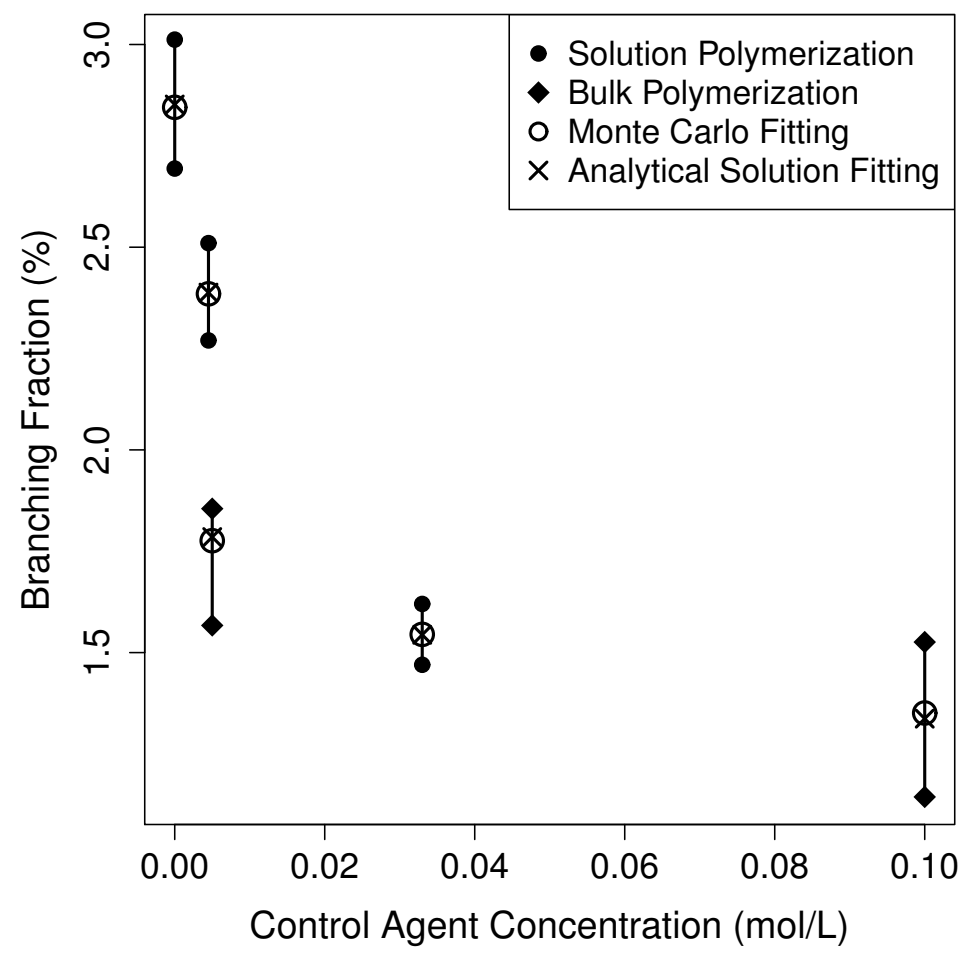

Figure 3: Fitted data obtained by the analytical approach (crosses) and by the MC method (open circles) (MC sample size $G=10^{4}$ ) are presented for two polymerization reactions, bulk and solution. Both approaches use the linear exponential inter-events times pdf (20).

\begin{tabular}{|c|c|c|c|c|c|c|c|}
\hline Polymerization & Fitting & $b_{p}$ & $b_{r}$ & $b_{d}$ & $\tau_{p}$ & $\tau_{r}$ & $\tau_{d}$ \\
\hline Solution & $\mathrm{AS}$ & $1.74 \times 10^{-1}$ & 6.53 & $2.28 \times 10^{-4}$ & $9.1 \times 10^{-1}$ & 1.31 & $3.58 \times 10^{-2}$ \\
\hline Solution & $\mathrm{MC}$ & $1.74 \times 10^{-1}$ & 6.53 & $2.28 \times 10^{-4}$ & $9.1 \times 10^{-1}$ & 1.31 & $3.58 \times 10^{-2}$ \\
\hline Bulk & $\mathrm{AS}$ & $2.8 \times 10^{-1}$ & $1.58 \times 10^{-1}$ & $1.57 \times 10^{-2}$ & $8.53 \times 10^{-1}$ & 11.54 & $3.43 \times 10^{-2}$ \\
\hline Bulk & $\mathrm{MC}$ & $1.64 \times 10^{-1}$ & $1.40 \times 10^{-1}$ & $3.557 \times 10^{-2}$ & $9.16 \times 10^{-1}$ & 12.01 & $2.56 \times 10^{-2}$ \\
\hline
\end{tabular}

Table 1: Optimized parameters of linear exponential inter-events times pdf (20) obtained by analytical solution fitting (AS) and Monte Carlo fitting (MC). All results assume unitary concentrations of monomers and control agents (supporting information can be found in Ref. [12]). 


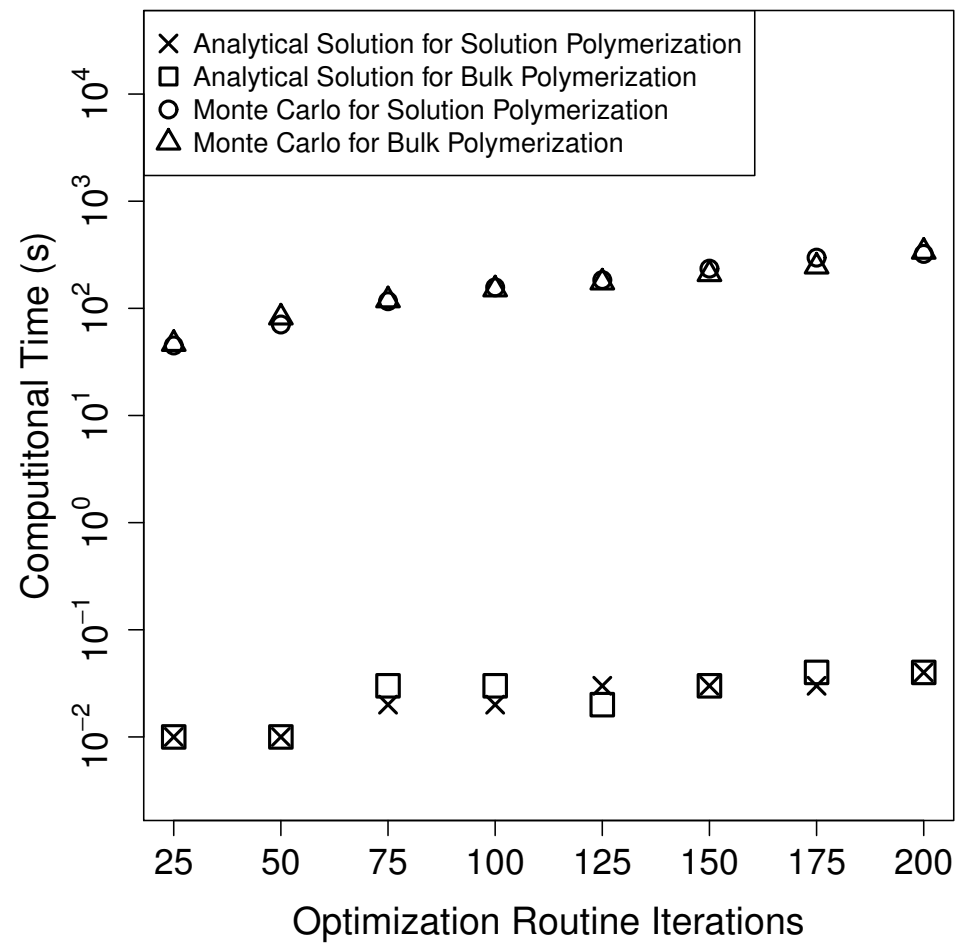

Figure 4: Computational times required for the optimization routine (Nelder-Mead method [15]) performed with an increasing number of iterations for bulk and solution polymerization. The analytical approach (crosses and squares) speeds up the procedure by the factor of $10^{4}$ compared with the MC based optimization method (open circles and triangles) of the same level of accuracy. 


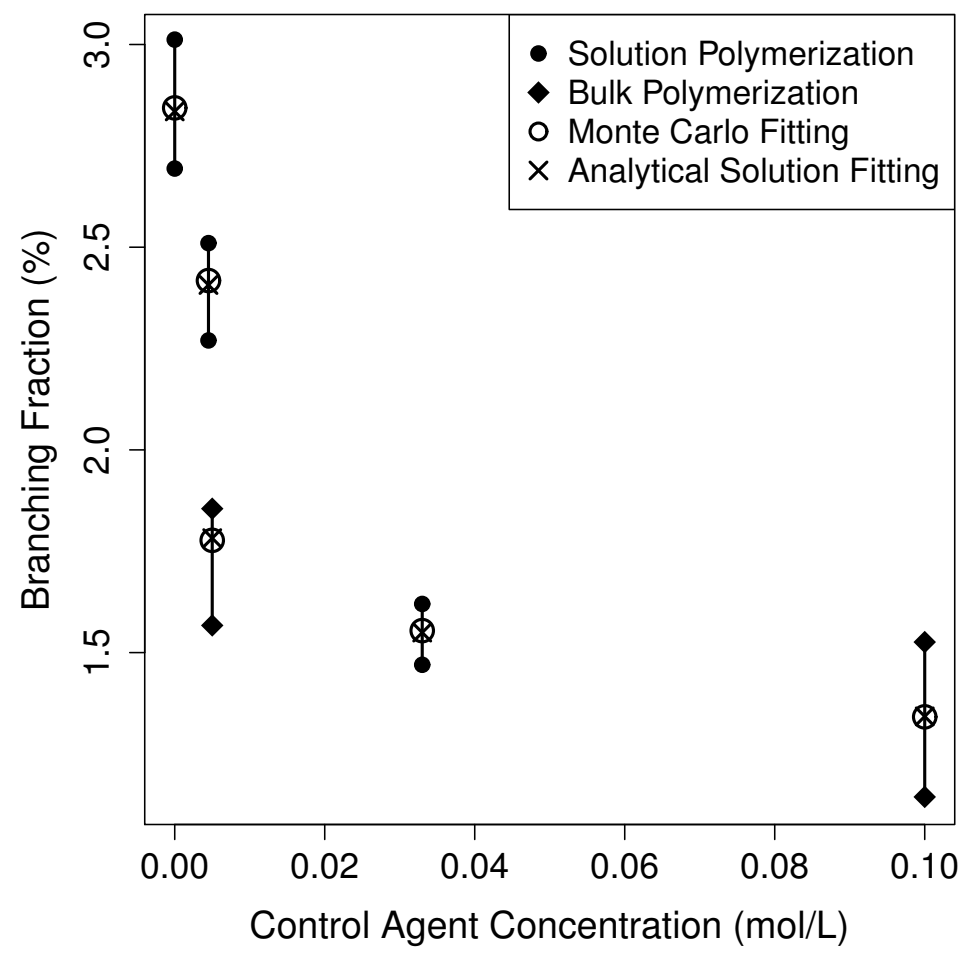

Figure 5: Fitted data obtained by the analytical approach (crosses) and by the MC method (open circles) (MC sample size $G=10^{4}$ ) are presented for two polymerization reactions, bulk and solution. Both approaches use exponential pdf's for propagation and deactivation, and a linear exponential pdf (20) for backbiting.

\begin{tabular}{|c|c|c|c|c|c|c|}
\hline Polymerization & $b_{p}$ & $b_{r}$ & $b_{d}$ & $\tau_{p}$ & $\tau_{r}$ & $\tau_{d}$ \\
\hline Solution & - & 5.94 & - & 1 & 5.97 & $4.78 \times 10^{-2}$ \\
\hline Bulk & - & $3.82 \times 10^{-1}$ & - & 1 & 8.80 & 1.33 \\
\hline
\end{tabular}

Table 2: Optimized parameters corresponding to the model with exponential pdf's for propagation and deactivation, and a linear exponential pdf (20) for backbiting. In the case of exponential pdf, the parameter $\tau$ expresses the mean value of the corresponding random variable. All results are for unitary concentrations of monomers and control agents (supporting information can be found in Ref. [12]). The shown set of parameters was found with the analytical solution fitting. Then, the same set was tested with the MC method, providing the data fitting shown in Figure 5. 


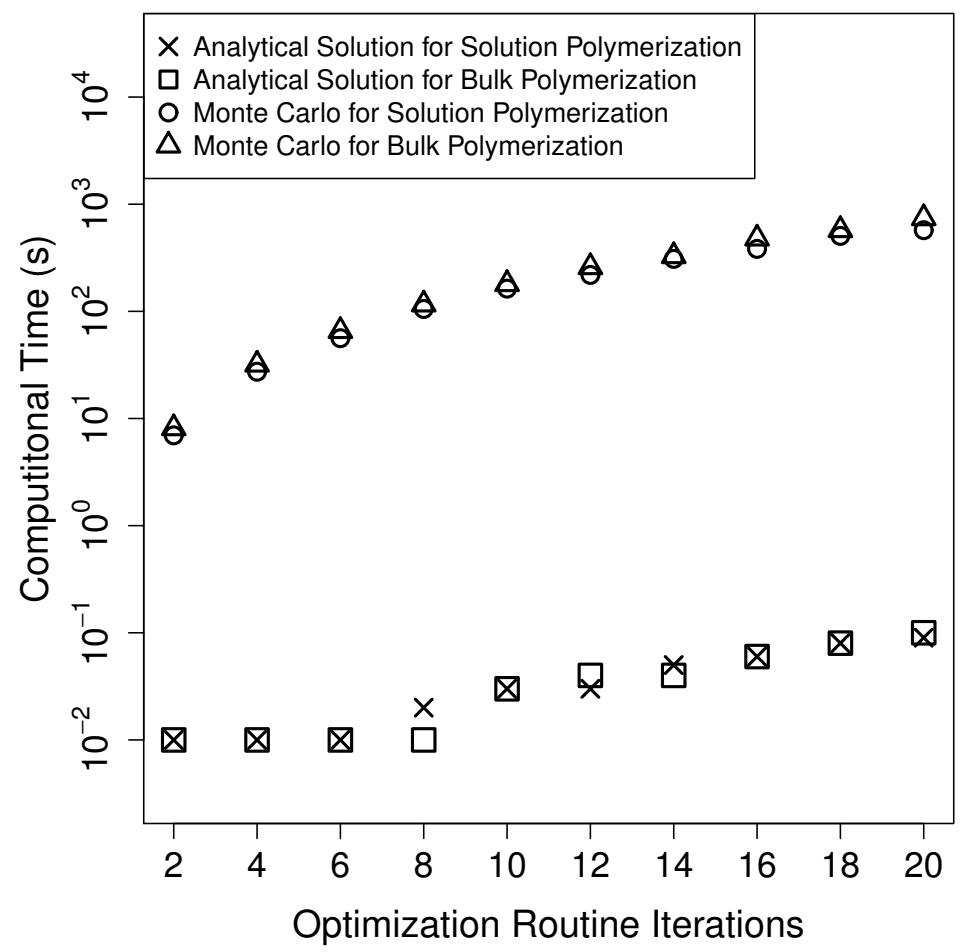

Figure 6: Computational times required for the optimization routine (Genetic Algorithm [16]) performed with an increasing number of iterations for bulk and solution polymerization. The analytical approach (crosses and squares) speeds up the procedure by the factor of $10^{4}$ compared with the MC based optimization method (open circles and triangles) of the same level of accuracy. 


\section{Conclusions}

Stochastic simulation algorithms produce multiple realizations of the full evolution of studied processes. In principle, this information allows a systematic study of all properties of the simulated system. The price for this detailed description includes, however, excessive computational time, needed to perform the Monte Carlo simulations, as well as the presence of unavoidable statistical errors. If the focus is on extracting particular quantities of interest, it may be possible to solve the problem analytically. The proposed analytical approach is one example of this strategy. We have demonstrated superiority of this analytical approach, over a traditional Monte Carlo simulation in the computation of the branching fraction in Controlled Radical Polymerization. The approach is free of statistical errors, and thus guarantees more accurate estimations, than those provided by a Monte Carlo simulation. In addition, the method is significantly (an order of the sample size) faster than the Monte Carlo approach. The performed tests show that the choice of optimization algorithm is not important and the analytical approach works with any choice of the inter-events time pdf. The proposed method is also general enough to be used in other applications, especially in those involving constrained stochastic processes which are difficult and often impossible to simulate using a conventional Statistical Simulation Algorithm. Finally, the proposed approach can be used as an efficient tool for finding the optimal set of parameters for inter-events time pdf, to be further utilized in detailed Monte Carlo simulations.

\section{Acknowledgements}

This research is supported by the Spanish Ministry of Economy and Competitiveness MINECO: BCAM Severo Ochoa accreditation SEV-2013-0323, grant SVP-2014-068451. One of us (EA) acknowledges the support of the project MTM2013-46553-C3-1-P. DS acknowledges support of the Basque Government (Grant No. IT-472-10), and of the Ministry of Science and Innovation of Spain (Grant No. FIS2009-12773-C02-01). The authors thank J.M. Asua (POLYMAT, Spain) for the valuable discussions.

\section{A Computation of the Probabilities}

Below we explain how to compute the probability $\mathbb{P}\left(T_{k}<T_{j} \forall j \neq k\right)$, with $k, j \in\{1, . ., N\}$. $T_{1}, . ., T_{N}$ are independent random variables with the generic pdf $f_{i}(t), i \in\{1, . . N\}$ :

$$
T_{i} \stackrel{\text { ind. }}{\sim} f_{i}(t) \quad \forall i \in\{1, . ., N\}
$$

The cumulative density function $F_{i}(t)$ of the random variable $T_{i}$ is the following:

$$
F_{i}(t):=\mathbb{P}\left(T_{i} \leq t\right)=\int_{0}^{t} f_{i}(\tau) d \tau \quad \forall i \in\{1, . ., N\} .
$$

The probability of interest is then computed as:

$$
\begin{aligned}
\mathbb{P}\left(T_{k}<T_{j} \forall j \neq k\right) & =\int_{0}^{+\infty} \mathbb{P}\left(T_{k}<T_{j} \forall j \neq k, T_{k}=\tau\right) d \tau= \\
& =\int_{0}^{+\infty} \mathbb{P}\left(T_{k}=\tau\right) \prod_{j \neq k} \mathbb{P}\left(T_{j}>\tau\right) d \tau= \\
& =\int_{0}^{+\infty} f_{k}(\tau) \prod_{j \neq k}\left(1-F_{j}(\tau)\right) d \tau \quad \forall k \in\{1, . ., N\} .
\end{aligned}
$$




\section{References}

[1] D. Gillespie. Exact stochastic simulation of coupled chemical reactions. Journal of Physical Chemistry, 81(25):2340-2361, 1977.

[2] A.B. Bortz, M.H. Kalos, and J. L. Lebowitz. A new algorithm for Monte Carlo simulation of Ising spin systems. Journal of Computational Physics, 17(1):10-18, 1975.

[3] D. Gillespie. Stochastic Simulation of Chemical Kinetics. Annu. Rev. Phys. Chem., 58:35$55,2007$.

[4] Y.T. Yeh, L. Yang, M. Watson, N.D. Goodman, and Pat Hanrahan. Synthesizing open worlds with constraints using locally annealed reversible jump MCMC. Journal ACM Transactions on Graphics (TOG), 31(4):4-56, 2012.

[5] N.M. Ahmad, B. Charleux, C. Farcet, C.J. Ferguson, S.G. Gaynor, B.S. Hawkett, F. Heatley, B. Klumperman, D. Konkolewicz, P.A. Lovell, K. Matyjaszewski, and R. Venkatesh. Chain Transfer to Polymer and Branching in Controlled Radical Polymerizations of n-Butyl Acrylate. Macromol. Rapid Commun., 30:2002-2021, 2009.

[6] Y. Reyes, G. Arzamendi, J.M Asua, and J.R. Leiza. Branching at High Frequency Pulsed Laser Polymerizations of Acrylate Monomers. Macromolecules, 44:3674-3679, 2011.

[7] C. Barner-Kowollik, M. Buback, B. Charleux, M.L. Coote, M. Drache, T. Fukuda, A. Goto, B. Klumperman, A.B. Lowe, J.B. Mcleary, G. Moad, M.J. Monteiro, R.D. Sanderson, M.P. Tonge, P. Vana, and P. Marie. Mechanism and Kinetics of Dithiobenzoate-Mediated RAFT Polymerization. I. The Current Situation. J. Polym. Sci., Part A: Polym. Chem., 44:58095831, 2006.

[8] G. Moad. Mechanism and Kinetics of Dithiobenzoate-Mediated RAFT Polymerization Status of the Dilemma. Macromol. Chem. Phys., 215:9-26, 2014.

[9] J.F.J. Coelho, A.C. Fonseca, P.M.F.O. Gonçalves, A.V. Popov, and M.H. Gil. Particle features and morphology of poly(vinyl chloride) prepared by living radical polymerisation in aqueous media. Insight about particle formation mechanism. Polymer, 52:2998-3010, 2011.

[10] G. Moad and D.H. Solomon. The Chemistry of Radical Polymerization. Elsevier, ISBN 0-08-044286-2, Second fully revised edition, 2006.

[11] D. Sokolovski, S. Rusconi, E. Akhmatskaya, and J.M. Asua. Non-Markovian models of the growth of a polymer chain. Proc. R. Soc. A, 471: 20140899.

[12] N. Ballard, S. Rusconi, E. Akhmatskaya, D. Sokolovski, J. de la Cal, and J.M. Asua. The Impact of Competitive Processes on Controlled Radical Polymerization. Macromolecules, 47(19):6580-6590, 2014.

[13] D.T. Gillespie, A. Hellander, and L.R. Petzold. Perspective: Stochastic algorithms for chemical kinetics. J. Chem. Phys., 138:170901-1-170901-14, 2013.

[14] N. Ballard, M. Salsamendi, J.I. Santos, F. Ruipérez, J.R. Leiza, and J.M. Asua. Experimental Evidence Shedding Light on the Origin of the Reduction of Branching of Acrylates in ATRP. Macromolecules, 47:964-972, 2014. 
[15] J.A. Nelder and R. Mead. A simplex method for function minimization. Computer Journal, $7(4): 308-313,1965$.

[16] M. Mitchell. An Introduction to Genetic Algorithms. Cambridge, MA: MIT Press, 1996. 